\title{
Genetic analysis of CPV vaccine strain NL-35-D and evaluation of induced neutralizing antibody against virulent wild-type CPV and FPV Shanghai isolates
}

Jianzhong Yi ( $\sim 2893898187 @ q q . c o m$ )

Shanghai Academy of Agricultural Sciences

Chengqian LiuX

Shanghai Academy of Agricultural Sciences

Hong Li

Shanghai Academy of Agricultural Sciences

Jun Gao

Shanghai Academy of Agricultural Sciences

Hongyu Liang

Shanghai Academy of Agricultural Sciences

Huili Liu

Shanghai Academy of Agricultural Sciences

Fengping Sun

Shanghai Academy of Agricultural Sciences

\section{Research Article}

Keywords: Genetic analysis, canine parvovirus, feline parvovirus, VP2 gene

Posted Date: August 27th, 2021

DOl: https://doi.org/10.21203/rs.3.rs-627692/v1

License: (9) This work is licensed under a Creative Commons Attribution 4.0 International License.

Read Full License 


\section{Abstract \\ Background}

Canine parvovirus (CPV) emerged in 1970s as a new causative disease of dogs worldwide. The virus was shown to be closely related, genetically and antigenically, to feline parvovirus (FPV) and to FPV-like parvoviruses from which were presumably originated by host species shift and subsequent rapid adaptation. CPV is the most important viral cause of enteritis and death in puppies, and vaccination is the most important and effective measure to prevent $\mathrm{CPV}$ infection. The live attenuated vaccine strain NL-35-D is widely used as a commercial vaccine in China and other countries, but its genome sequence has not been reported so far.

\section{Results}

We reported a near full length sequence of NL-35-D vaccine strain (Genbank: MW650831), and identified a CPV-SH2001 (Genbank: MW650830) and a FPV-SH2001 (Genbank: MW650831) isolates from the fecal samples of stray dogs and cats in Shanghai, respectively. Immunofluorescence assay revealed that the CPV and FPV isolates could be proliferated by F81 cells. we sequenced the full length genome of NL-35-D virus strain, phylogenetic analysis of full-length genome and VP2 gene in comparison with the CPV and FPV strains isolated worldwide. According to the genetic analysis of VP2 gene, the CPV-SH2001 and FPVSH2001 isolates were clustering far from the NL-35-D. However, the results demonstrated that NL-35-D vaccine strain still shares part of neutralizing epitopes with CPV and FPV isolates. The neutralizing antibody serum test also suggested vaccine strain NL-35-D is still effective against the present field isolated CPV and FPV strains. Therefore, these shared antigenic epitopes may be critical to the immune efficacy of the vaccine and deserve further testing and validation.

\section{Conclusions}

Our results revealed that pups inoculated with CPV vaccine had significantly high neutralizing antibody titers against $C P V$ and FPV virus strains. Pups vaccinated with CPV possess antibodies against heterologous viruses, the antibodies probably at levels still can provide complete or partial immunity in young pups.

\section{Background}

Canine parvovirus (CPV) and feline parvovirus (FPV), the major causative agent of acute hemorrhagic enteritis and myocarditis, are highly contagious and fatal pathogenic virus in dogs and cats (Appel et al., 1979; Kelly et al., 1978; Johnson et al., 1979). Since 1978 CPV was first recognized as an enteric pathogen of dogs, CPV viruses has spread throughout the world with high morbidity (100\%) and frequent mortality up to $10 \%$ in young puppies (Parrish et al., 1988;Greenwood et al., 1995). The close antigenic 
and genomic relationships among CPV, FPV and mink enteritis virus (MEV) (Parrish et al., 1991.) suggests that CPV may have originated in a wild host receptive to FPV by genetic mutation (Truyen et al., 1994; Zhou $P$ et al., 2017). Since the emergence of CPV in the late 1970s, due to rapid evolution, new CPV antigenic types were evolved as CPV-2a, CPV-2b (CPV2a with Asn426Asp), and CPV-2c (CPV2a with Asn426Glu) and completely replaced the original CPV virus strain (Buonavoglia et al., 2000; Greenwood et al., 1996; Sagazio et al.,1998). Commonly, CPV causes disease in un-vaccinated 1-6 month-old pups, $\mathrm{CPV}$ vaccine usually protected vaccinated pups from infection of field viruses, unless immunization failure due to the high level of maternally derived antibodies (MDAs)( Pollock et al., 1982), high-titer CPV vaccines and intranasal vaccine administration have been well applied to avoid of MDA interference (Pollock et al., 1982; Parrish CR, Carmichael LE.1983).

10 copies of VP1 and 60 to 70 copies of VP2, arranged as homo-or heterodimers, comprises the $22 \mathrm{~nm}$ parvovirus capsid (Tsao et al., 1991), the VP2 are exposed on the surface of the capsid and determine the antigenicity of CPV and FPV, which contains neutralizing epitopes, the clinical and immunological significance of the CPV antigenic variation is important question concerns (Martella et al., 2006), few studies have demonstrated any significant relevance of the antigenic changes with the ability of CPV vaccines to protect dogs from the infection of virulent wild-type CPV and FPV isolates (Appel, M. J. G., and L. E. Carmichael. 1987). Live attenuated virus vaccine strain NL-35-D currently is widely used as a commercial vaccine in china and other countries (Zhao et al.,2017; Zhuang QY et al., 2019; Li C et al., 2018). Vaccine strain NL-35-D are widely used in China, evaluation and monitoring of vaccine immune responses may help to determine the efficacy of the current vaccine, however, there is no reports about VP1, VP2 and full-length genome sequence of the vaccine strain NL-35-D, in the present study we sequenced the full length genome of NL-35-D virus strain, phylogenetic analysis of VP2 gene and full genome in comparison with the CPV and FPV virus strains isolated in china and in the world (Charoenkul $\mathrm{K}$ et al., 2019), and comparative analysis of the antigenic structure and variations of VP2 among vaccine strain and virulent wild-type CPV and FPV strains.

The aim of this study was to test the cross-reactivity of sera from pups inoculated with NL-35-D modified live virus vaccine strain and evaluate the serum neutralizing antibody titers against virulent wild-type CPV and FPV strains. HI assay and serum neutralization test (SNT) were used to evaluate the post-vaccine immune responses of CPV vaccinated pups.

\section{Results}

Confirmation of CPV and FPV in the fecal samples by rapid test and PCR

Fecal samples were diluted and then prepared for screening using both the TRFIA (Time resolved fluorescence immunoassay) test strip test and PCR. The positive samples are shown in Fig. 1, left panels of the figure show the result of the TRFIA test strip, the upper band is control band and the lower band is test band, the vaccine strain NL-35-D, fecal samples from sick dog and cat demonstrated positive results 
(Fig. 1, A). The PCR positive samples presented with a band at 1750 base pairs (bp) amplified using general test primers described previously (Fig. 1, B).

CPV and FPV virus isolation and proliferation

The cells were cultured in $37^{\circ} \mathrm{C}$ incubator containing $5 \% \mathrm{CO}_{2}$. After 4 passages of blind passage, the cytopathy was as high as $75-80 \%$. The cells in the control group showed complete boundary and uniform morphology. They are named CPV-SH2001 and FPV-SH2001 respectively. There are typical cytopathic morphological changes in CPV or FPV infected cells showing extensive regions of cell detachment (Fig. 2). The isolated CPV-SH2001 and FPV-SH2001 virus strains have the ability to infect and proliferate in F81 cells which was further proved by immunofluorescence assay (Fig. 2).

Full length genome phylogenetic analysis of live attenuated vaccine strain NL-35-D and virulent wild-type CPV and FPV isolates

Amplification and sequencing of the complete genomes (Fig. 3), encompassing both NS1, NS2 and VP1,VP2 genes, sequences were assembled by using BioEdit ver 7.2.5 software basing on overlapping strategy, Assembled nucleotide sequences are compared with those of CPV and FPV reference strains collected in China and in the world. Genomic phylogenetic analysis was performed using ML method by MEGA5 software. The vaccine strain NL-35-D has near full length genome of $4578 \mathrm{nt}$ and virulent wildtype CPV-SH2001 and FPV-SH2001 isolates have genomes of $5005 \mathrm{nt}$ and $5051 \mathrm{nt}$ in length and contain the ORFs: NS1 starts at position 267 and ends at position 2270 of the genome and encodes a protein of 668 amino acids; VP1 is generated by the alternative splicing of the fragment located at positions 22802308 and the fragment at positions $2381-4535$, which encodes a 727 amino acids protein; the VP2 starts at position 2781 and ends at position 4535 of the genome encoding a 668 amino acids protein.

The vaccine strain NL-35-D showed a well separated clade from FPV and CPV isolates sequences, between the FPV cluster and CPV clusters (Fig. 4). The FPV-SH2001 has cluster of FPV isolates from china, the CPV-SH2001 has clusters of CPV-2c isolates, CPV-2a and CPV-2b respectively clustered in the same phylogroup. Our analysis indicated that FPV and CPV showed a completely different pattern of evolution, although they are highly related at the genetic level. FPV has not experienced major changes in antigenic and biological properties, however CPV evolution leads to the emergence of new antigenic variants (2a, 2b and 2c). In this study, the near-complete genomes of NL-35-D, CPV and FPV strains is great value to further exploring antigenic variation between CPV and FPV strains and evaluating vaccine strain's efficiency.

VP2 gene phylogenetic analysis of NL-35-D and virulent wild-type CPV and FPV isolates

We used 29 VP2 sequences of virulent wild-type CPV and FPV selected from the Genbank for the analysis, and constructed phylogenetic tree by maximum likelihood method to determine the evolutionary relationship of the analyzed NL-35-D strain with other CPV and FPV strains from different geological areas(Fig. 5). The vaccine strain NL-35-D, much close to FPV clade than CPV-2b and CPV-2a clades. This 
tree indicated that phylogroups are generally formed as distinct species and different regions, some clusters are formed by regional strains, FPV isolates formed clusters exclusively, the close clustering of these isolates suggests that FPV is highly conserved. CPV isolates mainly formed three clusters CPV-2a, CPV-2b, CPV-2c, whereas, the CPV-2a strain (MT447096) lonely formed one clade,far away from other CPV-2a clades, this observation suggest this strain has evolved locally in Australia. The FPV strain MN270937 and CPV 2c strain MN270948 formed one clade, far away from other FPV and CPV clusters, this observation suggest the FPV and CPV-2c might co-infected host animals, and happened to gene recombination in Thailand.

Comparative analysis of VP2 protein

The major determinant antigenic epitopes of parvoviruses is on the VP2 protein, some amino acid residues influence the antigenicity and host range of both CPV and FPV strains, mutations at resides 80(K), $93(\mathrm{~K}), 103(\mathrm{~V}), 323(\mathrm{D}), 564(\mathrm{~N})$ and 568(A) will affect the antigenic characteristics and host range. Our analysis indicated that FPV has evolved mainly by random genetic drift without change of amino acid. The VP2 proteins of vaccine strain NL-35-D and FPV have the same amino acid at 5, 87, 267, 297, $300,305,324,370,426,440$. Whereas, the VP2 proteins of vaccine strain NL-35-D and CPV have the same amino acid at $80,93,103,232,323,564,568$. There are four linear neutralizing epitope on VP2 protein, at amino acid residues 2-16 (SDGAVQPDGGQPAVR), 91-99 (AVNGNMALD), 292-297 (NSLPQSS) and 297-309 (SEGATNFDGVE) (J P Langeveld et al., 1993); two dominant conformational epitopes, site A(composed of loop1, loop2, loop4) (Strassheim ML et al.,1994), whereas site B composed of only loop3. Vaccine strain NL-35-D respectively shares part of neutralizing epitopes with CPV and FPV strains, these observations suggests vaccine strain NL-35-D can induce neutralizing antibody against both viruses (Table 2).

The hemagglutination inhibition test (HI test)

In the present study, the $\mathrm{HI}$ test results were carried out using the sera of pups inoculated with CPV2 NL35-D, the $\mathrm{HI}$ titers of serum against CPV and FPV is about 1:256 (Fig. 6).

SNT test

The indirect immunofluorescence assay (IFA) test show that vaccine strain NL-35-D can induce neutralizing antibody against both CPV and FPV viruses, effectively protect F81cells from the infection of both viruses (Fig. 7).

\section{Discussion}

Since CPV appeared, evolutionary DNA changes have occurred within the viral genome,producing of new CPV variants, involving the substitutions in structural amino acid of virus capsid, and resulting in antigenic changes in each variants(Alfano et al., 2020). After 1980 the new variant CPV-2a almost 
completely replacement of $C P V-2$, the subsequent variants $C P V-2 b$ and $C P V-2 c$ also rapidly spread between dog and cat populations in the world due to the new genetic and phenotypical characteristics. New variants have the capability of adapting to different wild and domestic host animals(Ogbu et al., 2020). Severe clinical signs have been reported relating to CPV-2c in domestic cats, the CPV-2a and CPV$2 \mathrm{~b}$ variants also can replicate in cats $\triangle \mathrm{FPV}$ and $\mathrm{CPV}$ coinfections may occured in young kittens(Mara Battilani et al., 2011). Effective vaccine inoculation is great value in the prevention of CPV infection[virus (CPV) with the original antigenic structure are presently used to produce the licensed CPV vaccines $\square$ modified live $C P V$ vaccines have been immunized pups efficiently(Kelman $M$ et al., 2020), after virus challenge clinical ill signs couldn't be observed, no virus was recovered from fecal specimens(Vila Nova B et al., 2018;Cavalli A et al., 2020). Live attenuated vaccine strain NL-35-D currently is widely used as a commercial vaccine in china and other countries, we sequenced the full length genome of NL-35-D virus strain, phylogenetic analysis of full-length genome and VP2 gene in comparison with the CPV and FPV strains isolated worldwide. The genome phylogenetic analysis of CPV NL-35-D showed a separated clade in CPV clusters, between the FPV and CPV clusters.

VP2 has an important role in determination of antigenicity of CPV and FPV, there are four linear neutralizing epitope and two dominant conformational epitopes on VP2 protein. This study compare the antigenic structure and variations of VP2 among vaccine strain and virulent wild-type CPV and FPV strains, the results indicated that vaccine strain NL-35-D shares part of neutralizing epitopes with CPV and FPV respectively. Evaluation and monitoring of the immune responses induced with CPV vaccine may help to determine the efficiency of the present vaccine strain. The serological tests are presently used for diagnosis and decide the clinical significance of CPV antigenic biotype, since correlating with the immunity level of CPV and FPV, serum antibody titers are presently measured with hemagglutination inhibition (HI) test using CPV or FPV as the antigen which is employed by most investigators. Serum neutralization test (SNT) test is also standardly employed to measure the neutralizing antibody level in $\mathrm{CPV}$ vaccine inoculated dogs. This study also aimed to evaluate and monitor the post-vaccine immune responses in pups vaccinated by NL-35-D strain, $\mathrm{HI}$ assay and SNT assay were applied to measure the neutralizing antibody titer levels induced by vaccine strain, to evaluate the efficacy of NL-35-D strain in protecting against the infection of virulent field CPV and FPV isolates. Our results revealed that pups inoculated with CPV vaccine had significantly high neutralizing antibody titers against CPV and FPV virus strains. Pups vaccinated with CPV possess antibodies against heterologous viruses, the antibodies probably at levels still can provide complete or partial immunity in young pups.

\section{Conclusion}

We reported a near full length sequence of NL-35-D vaccine strain (Genbank: MW650831), and identified a CPV-SH2001 (Genbank: MW650830) and a FPV-SH2001 (Genbank: MW650831) isolates from the fecal samples of stray dogs and cats in Shanghai. The vaccine strain NL-35-D can induce neutralizing antibody against both CPV and FPV viruses, effectively protect F81 cells from the infection of both viruses. 


\section{Methods}

Specimens

Fecal specimens were obtained from one pet hospital located in Minhang district of Shanghai which is aimed at surveying animal infection diseases in china. All the samples were obtained with the owners consent and were collected following the recommendations in the Guide for the Care and Use of Laboratory Animals of the Ministry of Science and Technology of the People's Republic of China. Fecal samples were collected from one domestic cat and one domestic dog that had clinical signs of suspected parvovirus infection in shanghai.

Detection and confirmation of CPV and FPV

TRFIA (Time resolved fluoroimmunoassay) test strip (made by our laboratory) to diagnose FPV and CPV. Fecal samples from suspected dogs and cats were mixed with PBS, then the supernatants were added to TRFIA test strips at room temperature.

Viral DNA extraction and amplification

The Virus DNA extraction Kits (Takara Biotechnology) were used to extract total viral DNA from fecal samples according to the manufacturer's instructions. The extracted DNA was used for detection of CPV and FPV by polymerase chain reaction. CPV and FPV general detecting primers (PCR) using a primer pair (VP2-F:5'-ACAAGATAAAAGACGTGGTGTAACT-3'and VP2-R: 5'-ATGGGAAATACAGACTATAT-3'). The PCR reaction system including 1.0 $\mathrm{L}$ of template, 1.0 $\mu \mathrm{L}$ ach of VP2-F and VP2-R, 2×Ex Taq mix, and sufficient $\mathrm{ddH} 2 \mathrm{O}$ to increase the volume to $25 \mu \mathrm{L}$. Amplification was carried out as follows: one cycle at $95^{\circ} \mathrm{C}$ for 5 min; followed by 36 cycles at $95^{\circ} \mathrm{C}$ for $30 \mathrm{~s}, 58^{\circ} \mathrm{C}$ for $45 \mathrm{~s}$, and $72^{\circ} \mathrm{C}$ for $60 \mathrm{~s}$; and extension at $72^{\circ} \mathrm{C}$ for 5 min. PCR products were detected by electrophoresing using $12 \mu \mathrm{L}$ aliquots in $1.5 \%$ agarose gels in $1 \times \mathrm{TAE}$.

Cell cultivation and virus isolation

Samples were immersed in serum-free DMEM medium (Gibco), and clarified by centrifugation at 15.000 $\mathrm{rpm} / \mathrm{min}$ for $10 \mathrm{~min}$, then the supernatant was filtered through a $0.22 \mu \mathrm{m}$ Millipore filter (Millipore) and inoculated to F81 (feline kidney cell) cells (DMEM supplemented with $5 \%$ fetal bovine serum) at $37^{\circ} \mathrm{C}$ under $5 \% \mathrm{CO}_{2}$. The culture supernatants were harvested when the typical cytopathic effect (CPE) was observed almost $75 \%$ of the cells, subsequently, the culture medium was frozen at $-80^{\circ} \mathrm{C}$.

Immunofluorescence assay

F81 cells were seeded in 96-well plates, and the isolated CPV and FPV strains were respectively inoculated and incubated for $36 \mathrm{~h}$ at $37^{\circ} \mathrm{C}$ under $5 \% \mathrm{CO}_{2}$, removed the DMEM medium, washed the cells with phosphate buffered saline (PBST) for three rounds, fixed the cells with $75 \%$ ethanol for 15 min, incubated the primary antibody for $1 \mathrm{~h}$,then incubated with secondary antibody containing fluorescein 
isothiocyanate (FITC) for $1 \mathrm{~h}$ after washing the fixed cells by PBST for three cycles, Finally, a fluorescence inverted microscope was used to analyze fluorescence of the cells, normal F81 cells as negative controls.

Hemagglutinin test

The hemagglutination test was carried out using $1 \%$ porcine red blood cells. Four fold diluted virus was added to the V-type microplate containing red blood cells and stayed at $22^{\circ} \mathrm{C}$ for $45 \mathrm{~min}$. At least $50 \%$ red blood cells being hemagglutination was defined as positive result.

DNA purification and full length genome sequencing

The Virus DNA Kit (Takara Biotechnology) was used to extract virus DNA from the cell culture medium following the manufacturer's protocol. Full length genome Sequencing primer pairs were designed (Table 1). PCR amplification was generally achieved by 36 cycles of denaturation at $95^{\circ} \mathrm{C}$ for $30 \mathrm{~s}$, annealing at $58^{\circ} \mathrm{C}$ for $30 \mathrm{~s}$ and polymerization at $68^{\circ} \mathrm{C}$ for 3 min using KOD high fidelity DNA polymerase (Toyobo Biotechnology). the PCR products were excised from the gel and purified by a commercial kit (Sangon Biotech) after electrophoresis in a 1.5\% agarose gel and ethidium bromide staining, The PCR amplified DNA fragments were cloned into PMD18-T vector (Takara Biotechnology) for sequencing. The MegAlign tool (DNASTAR) was applied to assemble the nearly entire viral genome nucleotide sequences and the deduced amino acids sequence.

Phylogenetic analysis

Initially BLAST suite (blastn and blastx) was used to identify viral genome sequences through their similarity to CPV and FPV strains in Genbank, 24 near-full length genomes of carnivorous and feline parvoviruses were chosen for the analyses by the best hits of blastn search. Clustal X software was used to align all the genomes, and then construct a phylogenetic tree by the Maximum Composite Likelihood (MCL) approach, MEGAX software was used to conduct all the phylogenetic analyses and tree edition.

vaccine immunization and serum collection

A commercial multivalent vaccine (containing CPV vaccine strain NL-35-D) was used for vaccinating the pups with double booster. Blood samples were collected from all the vaccinated pups 14 days after second booster vaccination and then the collected blood samples were centrifuged and the serum was collected and stored at $-20^{\circ} \mathrm{C}$ until further testing.

HI test

$25 \mu \mathrm{L}$ of serum samples were diluted as two-fold serial dilutions in phosphate-buffered saline (PBS) (pH 7.0), then $4 \mathrm{HA}$ units of CPV vaccine strain was added to each well. The isolated field strains CPV$\mathrm{SH} 2001$ and FPV-SH2001 were respectively used for preparing $4 \mathrm{HAU}$. The plates were incubated at $22^{\circ} \mathrm{C}$ for $1 \mathrm{~h}$. Then, $50 \mu \mathrm{L}$ of $1 \%$ of porcine red blood cells were added with gentle mixing and left at $22^{\circ} \mathrm{C}$ for $1 \mathrm{~h}$. Cell controls was mixing $50 \mu \mathrm{L}$ of $0.2 \mathrm{M}$ Sorenson's PBS with $50 \mu \mathrm{L}$ of $1 \%$ of porcine red blood cells, virus 
controls was mixing $25 \mu \mathrm{L}$ of $0.2 \mathrm{M}$ Sorenson's PBS, $25 \mu \mathrm{L}$ of $4 \mathrm{HA}$ units of virus, and $50 \mu \mathrm{L}$ of $1 \%$ of porcine erythrocytes.

\section{SNT}

Serial fourfold dilutions (starting from 1:10) of each serum in DMEM were mixed with $50 \mathrm{ml}$ of a virus suspension containing 300 TCID50 of virus strain. After $1 \mathrm{~h}$ of incubation at room temperature, $20 \mathrm{ml}$ of a suspension containing 40.000 F81 cells was added to each well and incubated for 3 days, and virus was then detected using the IFA test as described above. Neutralizing antibody titer was calculated as the highest serum dilution which could completely neutralize the virus.

\section{List Of Abbreviations}

\section{List of abbreviations}

CPV(Canine parvovirus)

FPV (Feline parvovirus)

TRFIA (Time resolved fluoroimmunoassay)

HI(Hemagglutination inhibition)

IFA(The indirect immunofluorescence assay)

SNT(Serum neutralization test)

Table 2 Genetic analysis of VP2 amino acids of NL-35-D in comparison to CPV-SH2001,FPV-SH2001 and other different genotype CPV and FPV strains.

\section{Declarations}

\section{Ethics approval and consent to participate}

All the samples were collected following the recommendations in the Guide for the Care and Use of Laboratory Animals of the Ministry of Science and Technology of the People's Republic of China.The Animal Care and Use Committee of Shanghai Academy of Agricultural Science reviewed protocols, including operation details as well as approaches to ameliorate animal suffering and euthanasia.

\section{Consent for publication}

All authors consent for publication

\section{Availability of data and materials}

All data generated or analysed during this study are included in this published article. 


\section{Competing interests}

The authors declare no conflicts of interest.

\section{Funding}

This work was supported by the Shanghai Agricultural Applied Technology Development Program,China (Surveillance of major zoonosis in dogs and cats and risk assessment of public health safety in urban areas. project number: $2019-3-3$ )

\section{Authors' contributions}

\section{Acknowledgements}

JY designed the research.CL,HL,JG,HL,FS and HL performed the research.CL and JY wrote the paper and have contributed equally to this study

\section{References}

1. Appel, M. J. G., W. F. Scott, and L. E. Carmichael. 1979. Isolation and immunization studies of a canine parvo-like virus from dogs with haemorrhagic enteritis. Vet. Rec. 105:156-159.

2. Kelly, W. R. 1978. An enteric disease of dogs resembling feline panleukopaenia virus. Aust. Vet. J. 54:593.

3. Burtonboy, G., F. Coignoul, N. Delferriere, and P. P. Pastoret. 1979. Canine hemorrhagic enteritis: detection of viral particles by electron microscopy.Arch. Virol. 61:1-11.

4. Johnson, R. H., and P. B. Spradbrow. 1979. Isolation from dogs with severe enteritis of a parvovirus related to feline panleukopaenia virus. Aust. Vet. J.55:151.

5. Buonavoglia, C., M. Compagnucci, and Z. Orfei. 1983. Dog response to plaque variant of canine parvovirus. J. Vet. Med. B 30:526-531.

6. Parrish, C. R., P. H. O'Connel, J. F. Evermann, and L. E. Carmichael. 1988. Global spread and replacement of canine parvovirus strains. J. Gen. Virol.69:1111-1116.

7. Greenwood, N. M., W. S. Chalmers, W. Baxendale, and H. Thompson. 1995. Comparison of isolates of canine parvovirus by restriction enzyme analysis, and vaccine efficacy against field strains. Vet. Rec. 136:63-67.

8. Parrish, C. R., C. F. Aquadro, M. L. Strassheim, J. F. Evermann, J. Y. Sgro, and H. O. Mohammed. 1991. Rapid antigenic-type replacement and DNA sequence evolution of canine parvovirus. Virology 129:401414. 
9. De Ybanez, R. R., C. Vela, E. Cortes, I. Simarro, and Y. I. Casal. 1995. Identification of types of canine parvovirus circulating in Spain. Vet. Rec. 136:174-175.

10. Truyen, U., M. Agbandje, and C. R. Parrish. 1994. Characterization of the feline host range and a specific epitope of feline panleukopenia virus. Virology 200:494-503.

11. Zhou P, Zeng W, Zhang X, Li S. 2017. The genetic evolution of canine parvovirus - A new perspective. PLoS One. Mar 31;12(3).

12. Buonavoglia, D., A. Cavalli, A. Pratelli, V. Martella, G. Greco, M. Tempesta, and C. Buonavoglia. 2000. Antigenic analysis of canine parvovirus strains isolated in Italy. Microbiologica 23:93-96.

13. Greenwood, N. M., W. S. Chalmers, W. Baxendale, and H. Thompson. 1996. Comparison of isolates of canine parvovirus by monoclonal antibody and restriction enzyme-analysis. Vet. Rec. 138:495-496.

14. Sagazio, P., M. Tempesta, D. Buonavoglia, M. G. De Palma, and C. Buonavoglia. 1998. Antigenic relationship between CPV2 and CPV2b: results of a serological study, p. 43. Proceedings of the 1st International Meeting, Virology of Carnivores, Utrecht, The Netherlands.

15. Pollock, R. V. H., and L. E. Carmichael. 1982. Maternally derived immunity to canine parvovirus infection: transfer, decline, and interference with vaccination. J. Am. Vet. Med. Assoc. 180:37-42.

16. Pollock, R. V. H., and L. E. Carmichael. 1982. Dog response to inactivated canine parvovirus and feline panleukopenia virus vaccines. Cornell Vet.72:16-35.

17. Parrish CR, Carmichael LE. 1983. Antigenic structure and variation of canine parvovirus type-2, feline panleukopenia virus, and mink enteritis virus. Virology. Sep; 129(2):401-14.

18. Tsao J, Chapman MS, Agbandje M, Keller W, Smith K, Wu H, Luo M, Smith TJ, Rossmann MG, Compans RW, et al. 1991. The three-dimensional structure of canine parvovirus and its functional implications. Science. Mar 22; 251(5000):1456-64.

19. Martella V, Decaro N, Buonavoglia C. 2006. Evolution of CPV-2 and implicance for antigenic/genetic characterization. Virus genes. 33(1):11-13.

20. Appel, M. J. G., and L. E. Carmichael. 1987. Can a commercial vaccine protect pups against a recent isolate of canine parvovirus? Vet. Med. 10:1091-1093.

21. Zhao H, Wang J, Jiang Y, Cheng Y, Lin P, Zhu H, Han G, Yi L, Zhang S, Gu L, Cheng S. 2017. Typing of Canine Parvovirus Strains Circulating in North-East China. TransboundEmerg Dis. Apr; 64(2):495-503

22. Zhuang QY, Qiu Y, Pan ZH, Wang SC, Wang B, Wu WK, Yu JM, Yi Y, Sun FL, Wang KC. 2019. Genome sequence characterization of canine parvoviruses prevalent in the Sichuan province of China.

Transbound Emerg Dis. Mar; 66(2):897-907. 
23. Li C, Tang J, Chen Z, Li Q, Huang Z, Wang Q, Meng C, Wang Y, Liu G. 2018. Genetic characterization of the complete genome of a mutant canine parvovirus isolated in China. Arch Virol. Feb; 163(2):521-525.

24. Charoenkul K, Tangwangvivat R, Janetanakit T, Boonyapisitsopa S, Bunpapong N, Chaiyawong S, Amonsin A. 2019. Emergence of canine parvovirus type $2 c$ in domestic dogs and cats from Thailand. Transbound Emerg Dis. Jul; 66(4):1518-1528.

25. J P Langeveld, J I Casal, C Vela, K Dalsgaard, S H Smale, W C Puijk, R H Meloen. 1993. B-cell epitopes of canine parvovirus: distribution on the primary structure and exposure on the viral surface.J Virol.Feb;67(2):765-72.

26. Strassheim ML, Gruenberg A, Veijalainen P, Sgro JY, Parrish CR. 1994.Two dominant neutralizing antigenic determinants of canine parvovirus are found on the threefold spike of the virus capsid.Virology. Jan; 198(1):175-84.

27. Alfano F, Fusco G, Mari V, Occhiogrosso L, Miletti G, Brunetti R, Galiero G, Desario C, Cirilli M, Decaro N. 2020.Circulation of pantropic canine coronavirus in autochthonous and imported dogs, Italy. Transbound Emerg Dis. Mar 12:10.

28. Ogbu KI, Mira F, Purpari G, Nwosuh C, Loria GR, Schirò G, Chiaramonte G, Tion MT, Di Bella S, Ventriglia G, Decaro N, Anene BM, Guercio A. 2020.Nearly full-length genome characterization of canine parvovirus strains circulating in Nigeria. Transbound Emerg Dis. Mar; 67(2):635-647.

29هMara Battilani , Andrea Balboni, Martina Ustulin, Massimo Giunti, Alessandra Scagliarini, Santino

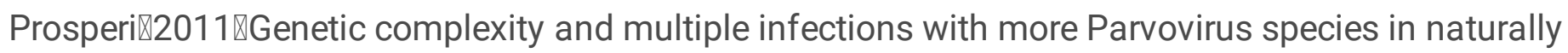
infected cats. Vet Res $₫$ Mar 2; 42(1)凹

30『Kelman M, Barrs VR, Norris JM, Ward MP. 2020区 Canine parvovirus prevention and prevalence: Veterinarian perceptions and behaviors.Prev Vet Med. Jan;174:104817.

31. Vila Nova B, Cunha E, Sepúlveda N, Oliveira M, São Braz B, Tavares L, Almeida V, Gil S. 2018】

Evaluation of the humoral immune response induced by vaccination for canine distemper and parvovirus: a pilot study.BMC Vet Res. Nov 16;14(1):348.

32. Wilson S, Illambas J, Siedek E, Stirling C, Thomas A, Plevová E, Sture G, Salt J. Vaccination of dogs with canine parvovirus type $2 b$ (CPV-2b) induces neutralising antibody responses to CPV-2a and CPV2c.Vaccine. 2014 Sep 22; 32(42):5420-4.

33. Cavalli A, Desario C, Marinaro M, Losurdo M, Camero M, Decaro N, Catella C, Lanave G, Buonavoglia C. 2020囚Oral administration of modified live canine parvovirus type $2 \mathrm{~b}$ induces systemic immune response. Vaccine. Jan 10; 38(2):115-118. 


\section{Tables}

Tabal 1 Full length genome Sequencing primer pairs

\begin{tabular}{llc}
\hline Name & Sequence(5' ${ }^{\prime}$ ' $\left.^{\prime}\right)$ & Product(bp) \\
\hline F1(205) & ATAGACCGTTACTGACATTCGCTTC & 1022 \\
R1(1226) & TCCTGGTTGTGCCATCATTTC & \\
F2(1044) & CCAGAAACCGTTGAAACCACAG & 937 \\
R2(1980) & GTATTTTAGGCTCCGCCCAGTT & \\
F3(1934) & GGGAAAAGTACCAGAATGGGATG & 974 \\
R3(2907) & TAGAAATCCCCACACCCCCAG & \multirow{2}{*}{1056} \\
F4(2778) & GCACCAATGAGTGATGGAGGAGT & \\
R4(3833) & TGTGTAGACGCCTCAAAAGAATAAT & \multirow{2}{*}{1209} \\
F5(3574) & GAACAGGTGATGAATTTGCTACAGG & \\
R5(4758) & CAACCACCCACACCATAAC & \\
\hline
\end{tabular}

Table 2 Genetic analysis of VP2 amino acids of NL-35-D in comparison to CPV-SH2001,FPV-SH2001 and other different genotype CPV and FPV strains.

\begin{tabular}{|c|c|c|c|c|c|c|c|c|c|c|c|c|c|c|c|c|c|c|c|}
\hline \multirow{2}{*}{ Genbank ID } & \multirow{2}{*}{ Genotype } & \multirow{2}{*}{ Name } & \multicolumn{17}{|c|}{ Amino acid site position on VP2 gene } \\
\hline & & & 5 & 80 & 87 & 93 & 103 & 232 & 267 & 297 & 300 & 305 & 323 & 324 & 370 & 426 & 440 & 564 & 568 \\
\hline MW650832 & CPV-2 & vaccine/NL-35-D & A & R & M & $\mathrm{N}$ & A & 1 & $\mathrm{~F}$ & s & A & D & $\mathrm{N}$ & r & $\mathrm{a}$ & $\mathrm{N}$ & T & $s$ & G \\
\hline MW650830 & CPV $-2 c$ & CPV-SH2001 & G & $\mathrm{R}$ & L & N & A & 1 & y & A & G & Y & $\mathrm{N}$ & 1 & $R$ & $E$ & T & $\mathrm{s}$ & G \\
\hline MN810882 & CPV-2a & CPV/LY-2 & A & R & L & $\mathrm{N}$ & A & 1 & y & A & G & r & $\mathrm{N}$ & 1 & $\mathrm{Q}$ & $\mathrm{N}$ & A & $\mathrm{N}$ & G \\
\hline MN810884 & CPV-2a & CPV/CC1-103 & A & $\mathrm{R}$ & L & $\mathrm{N}$ & A & 1 & y & A & G & Y & $\mathrm{N}$ & 1 & $\mathrm{a}$ & $\mathrm{N}$ & A & $\mathrm{N}$ & G \\
\hline MT488462 & CPV-2a & $\mathrm{CPV} / \mathrm{XH} 3 / 2018$ & A & $\mathrm{R}$ & L & $\mathrm{N}$ & A & 1 & y & A & G & Y & $\mathrm{N}$ & 1 & $\mathrm{Q}$ & $\mathrm{N}$ & A & $\mathrm{s}$ & $G$ \\
\hline MT488466 & $C P V-2 b$ & $\mathrm{CPV} / \mathrm{DH} 7 / 2018$ & A & R & L & $\mathrm{N}$ & A & 1 & y & A & G & Y & $\mathrm{N}$ & 1 & $\mathrm{Q}$ & D & T & s & G \\
\hline MH822028 & $C P V-2 b$ & CPV-2b/YZ1405 & A & $\mathrm{R}$ & L & $\mathrm{N}$ & A & 1 & y & A & G & Y & $\mathrm{N}$ & 1 & $Q$ & D & A & $\mathrm{s}$ & G \\
\hline MK517984 & CPV-2b & $\mathrm{CPV} / \mathrm{CN} / \mathrm{HN} 1719$ & A & $\mathrm{R}$ & L & $\mathrm{N}$ & A & 1 & y & A & G & r & $\mathrm{N}$ & 1 & a & D & A & 5 & G \\
\hline MN810876 & CPV $-2 \mathrm{C}$ & CPV/LY-8 & G & R & L & $\mathrm{N}$ & A & 1 & y & A & G & r & N & 1 & R & E & $\mathrm{T}$ & $\mathrm{s}$ & G \\
\hline MN810881 & CPV-2c & $\mathrm{CPV} / \mathrm{LY}-3$ & G & $\mathrm{R}$ & $\mathrm{L}$ & $\mathrm{N}$ & A & 1 & y & A & G & r & $\mathrm{N}$ & 1 & $\mathrm{R}$ & E & T & $\mathrm{s}$ & G \\
\hline MN119617 & $\mathrm{CPV}-2 \mathrm{C}$ & CPV/ CH-ZJ-D13 & $G$ & $\mathrm{R}$ & L & $\mathrm{N}$ & A & 1 & y & A & G & r & $\mathrm{N}$ & 1 & $\mathrm{R}$ & $E$ & $T$ & 5 & G \\
\hline MW650831 & & FPV-SH2001 & A & $\mathrm{k}$ & M & k & v & $v$ & $\mathrm{~F}$ & s & A & D & D & r & $\mathrm{Q}$ & $\mathrm{N}$ & $T$ & s & A \\
\hline MN270937 & & FPV-VT2020 & A & k & M & k & $\mathrm{v}$ & $\mathrm{v}$ & $\mathrm{F}$ & s & A & D & D & y & a & $\mathrm{N}$ & T & $s$ & A \\
\hline MK266788 & & FPV/ Guilyang01 & A & $\mathrm{k}$ & M & $\mathrm{k}$ & $v$ & $v$ & $\mathrm{~F}$ & s & A & D & D & y & $\mathrm{Q}$ & $\mathrm{N}$ & $\mathrm{T}$ & $\mathrm{N}$ & A \\
\hline МК266793 & & FPV/ Tanjin-01 & A & k & M & k & $v$ & $v$ & $\mathrm{~F}$ & s & A & D & D & y & Q & $\mathrm{N}$ & $T$ & $\mathrm{~N}$ & A \\
\hline MK266795 & & FPV/ Beijing-L3 & A & k & M & k & $\mathrm{v}$ & $\mathrm{v}$ & $\mathrm{F}$ & s & A & D & D & y & $\mathrm{a}$ & $\mathrm{N}$ & $T$ & $\mathrm{~N}$ & A \\
\hline
\end{tabular}


Figures
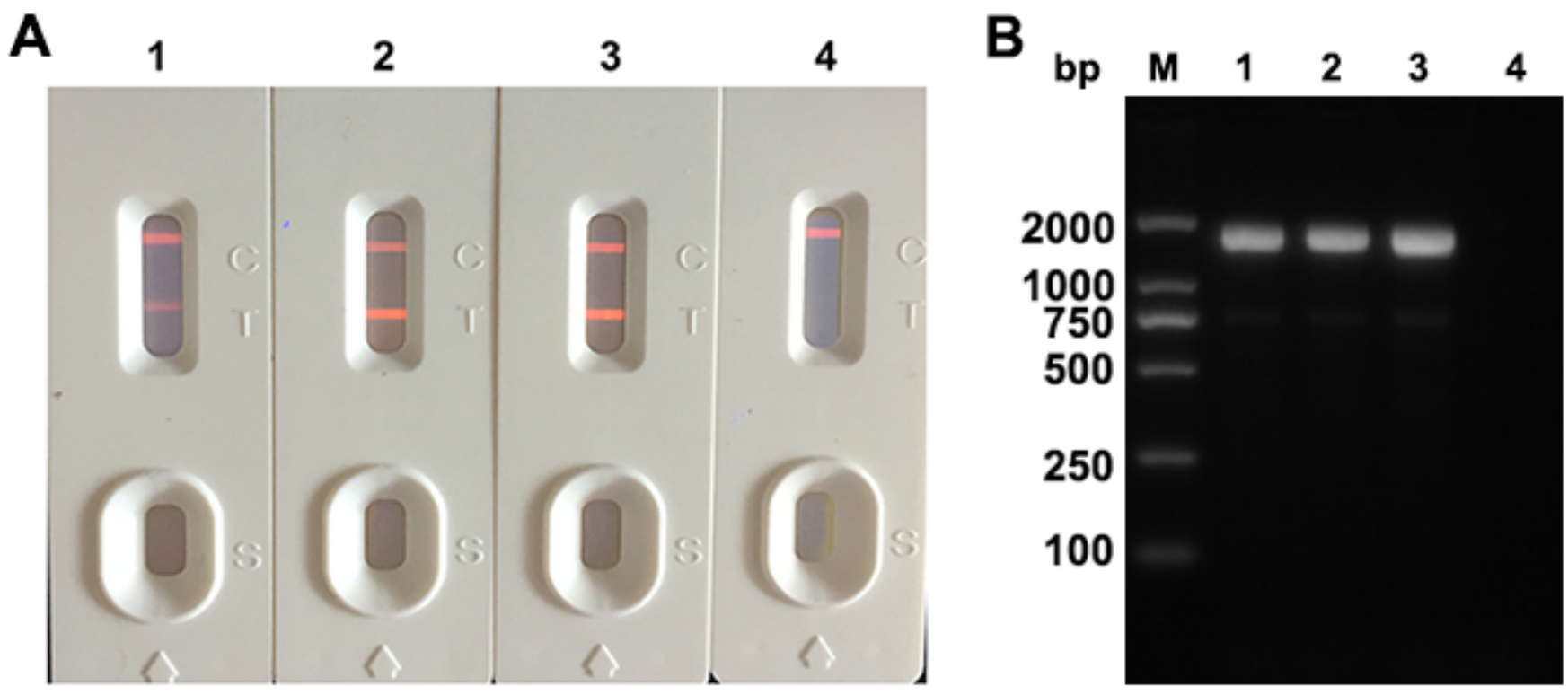

Figure 1

A ( TRFIA test): 1, vaccine strain NL-35-D; 2, CPV-SH2001; 3, FPV-SH2001; 4, negative control. B (amplification of CPV and FPV VP2 gene): M, Trans2K DNA Marker; 1, vaccine strain NL-35-D; 2, CPVSH2001; 3, FPV-SH2001; 4, negative control.
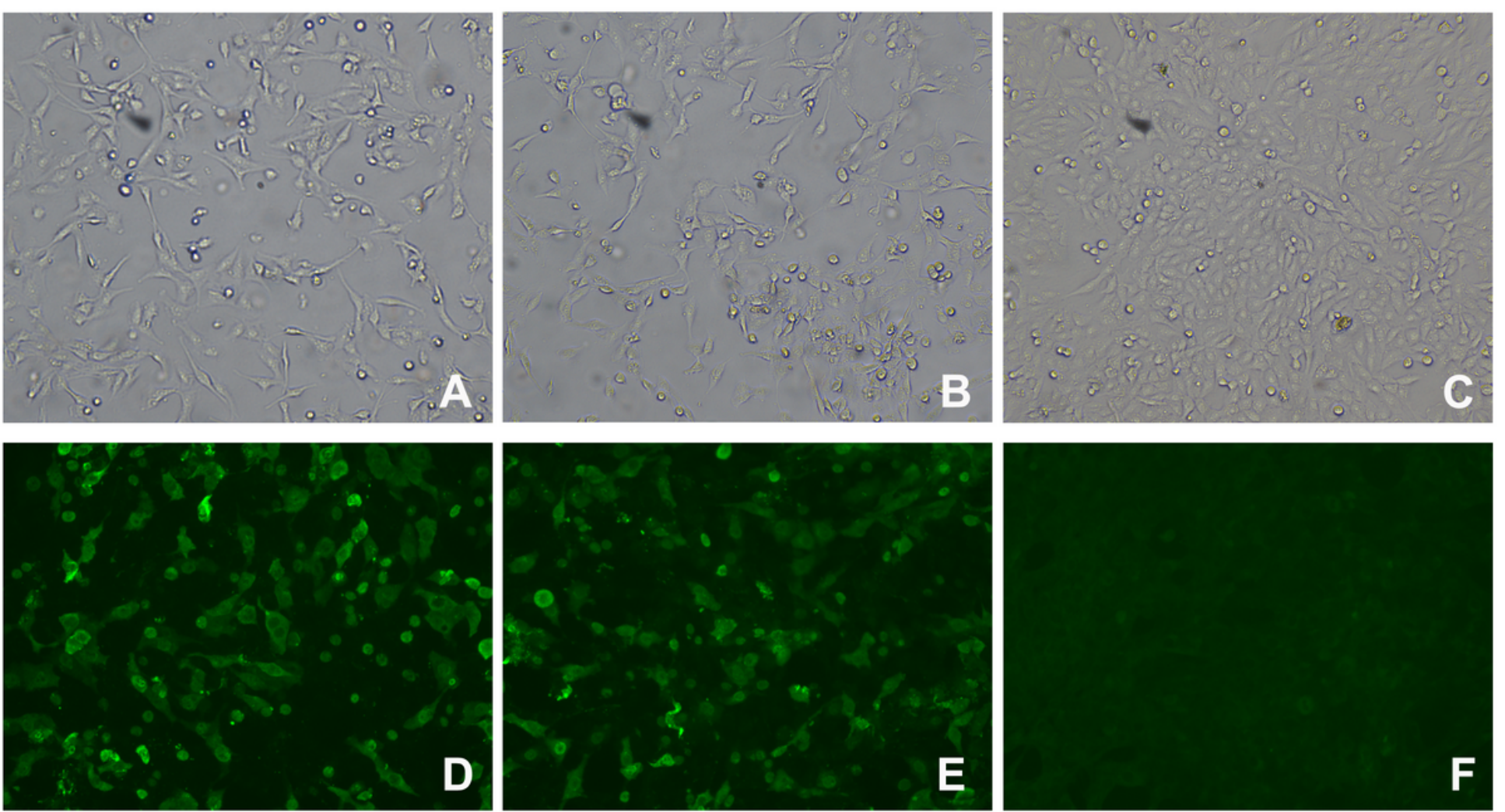

Figure 2 
The isolation and proliferation of CPV-SH2001 and FPV-SH2001 virus strain: A, CPV-SH2001; B, FPVSH2001; C, F81 cells control; the Immunofluorescence assay of isolated virus strains (400X): D, CPVSH2001; E, FPV-SH2001; F, F81 cells control.

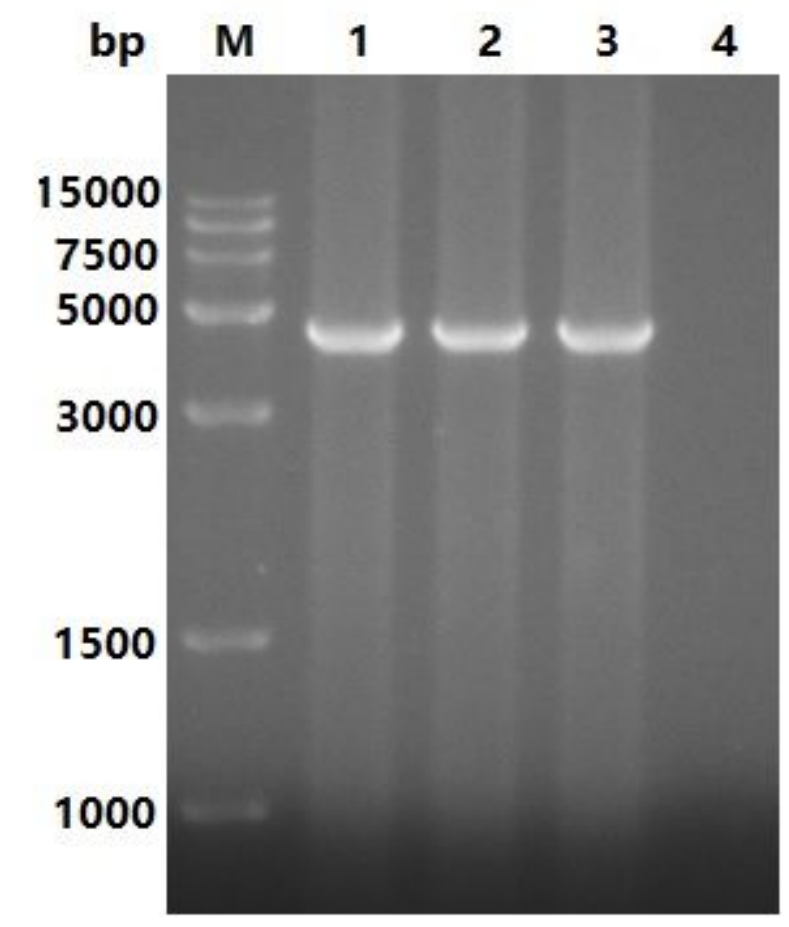

\section{Figure 3}

The full-length genome amplification. M, DNA Marker; 1, vaccine strain NL-35-D; 2, CPV-SH2001; 3, FPVSH2001; 4, negative control. 


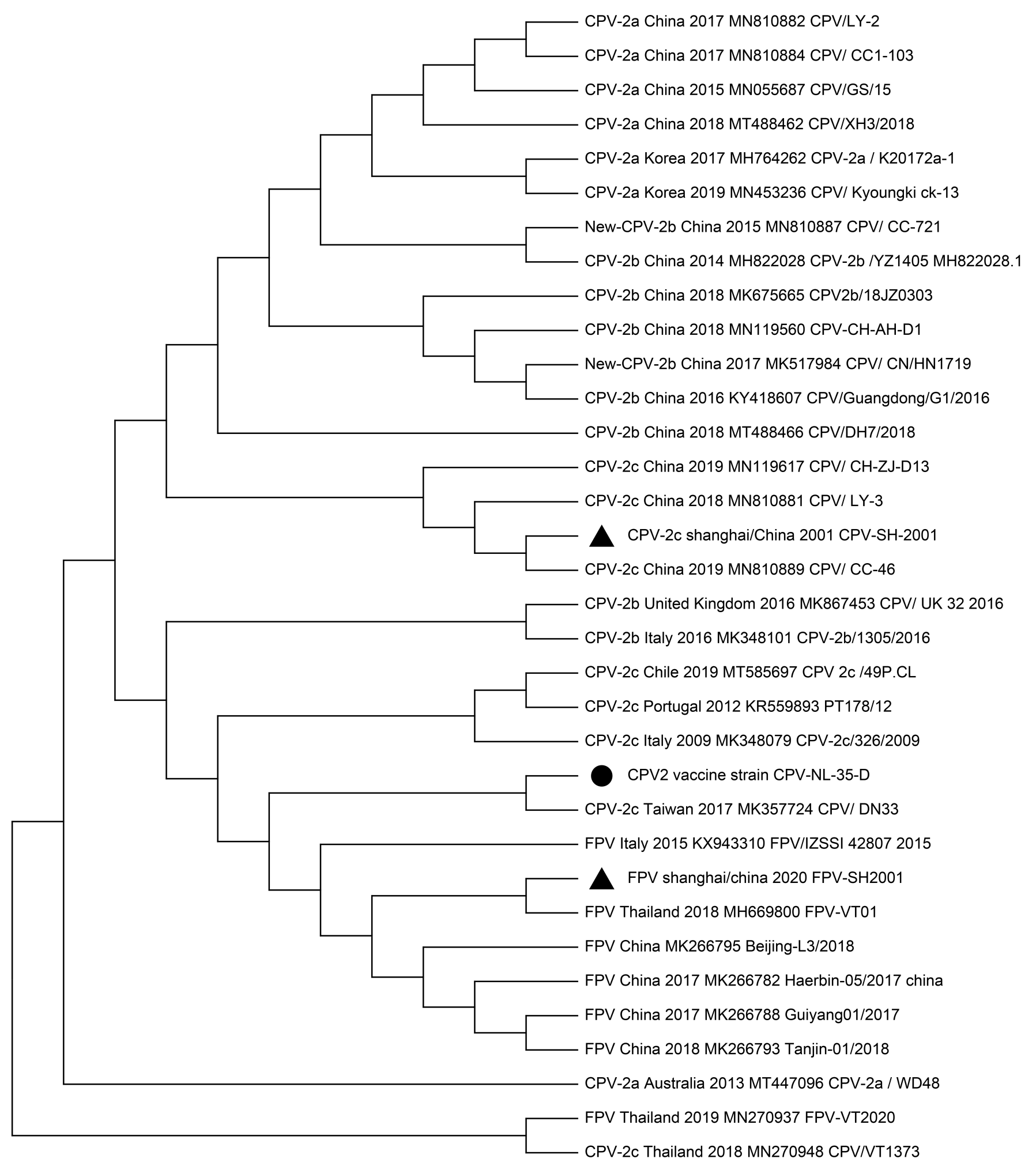

\section{Figure 4}

Maximum-likelihood tree based on full-length genome sequences of vaccine strain NL-35-D ( $)$, CPV-

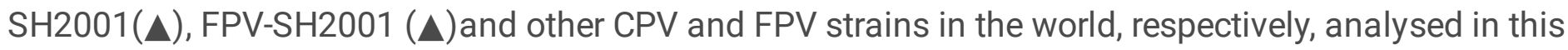
study. Each sequence is indicated with virus type (FPV, CPV, or variant CPV-2a, CPV-2b, CPV-2c), country and year of collection, accession number and strain/isolate name. 


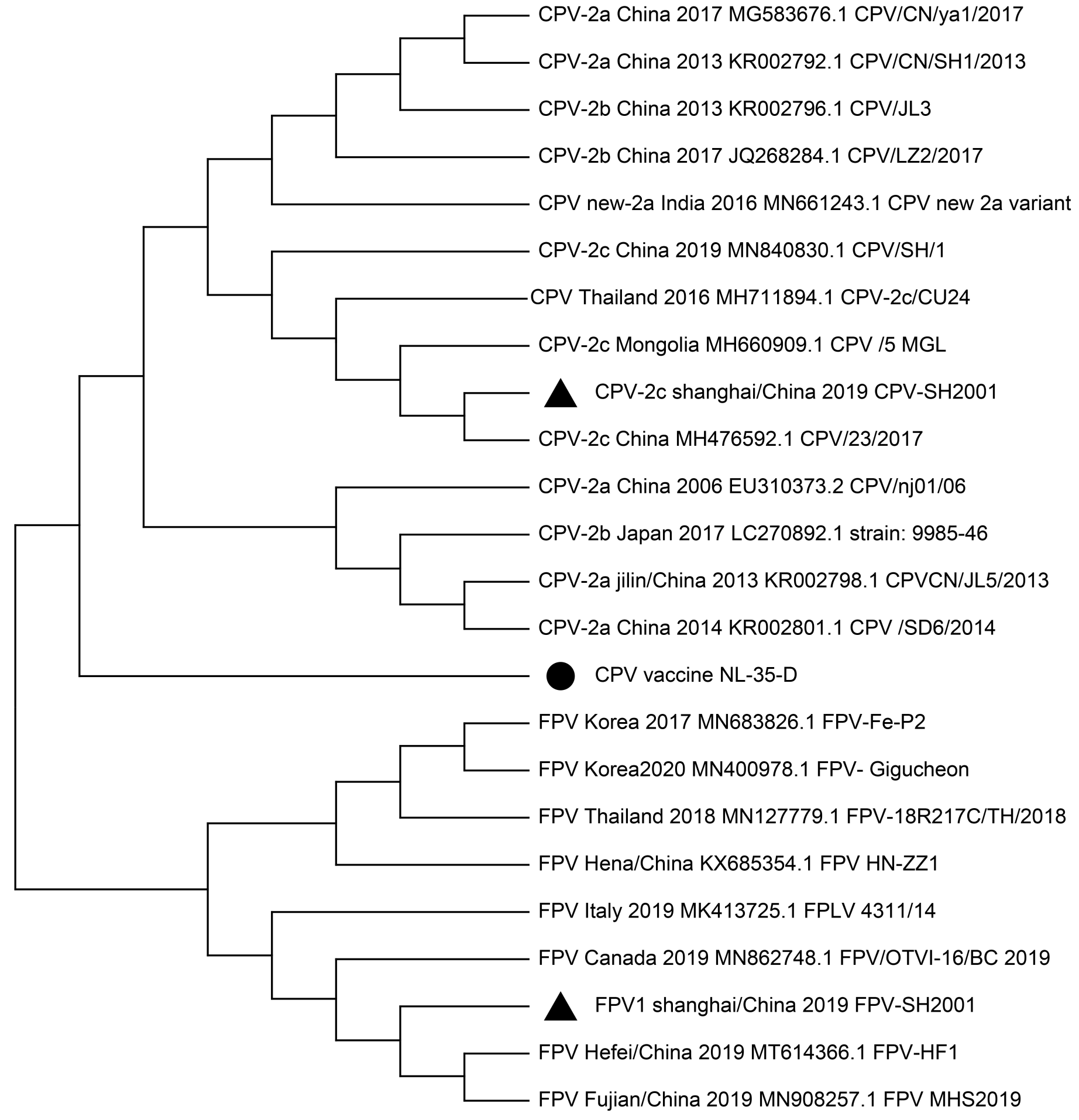

Figure 5

Maximum-likelihood tree based on full-length VP2 gene sequences of vaccine strain NL-35-D ( $)$, CPV-

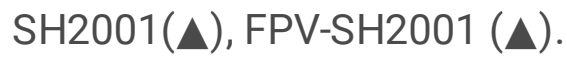




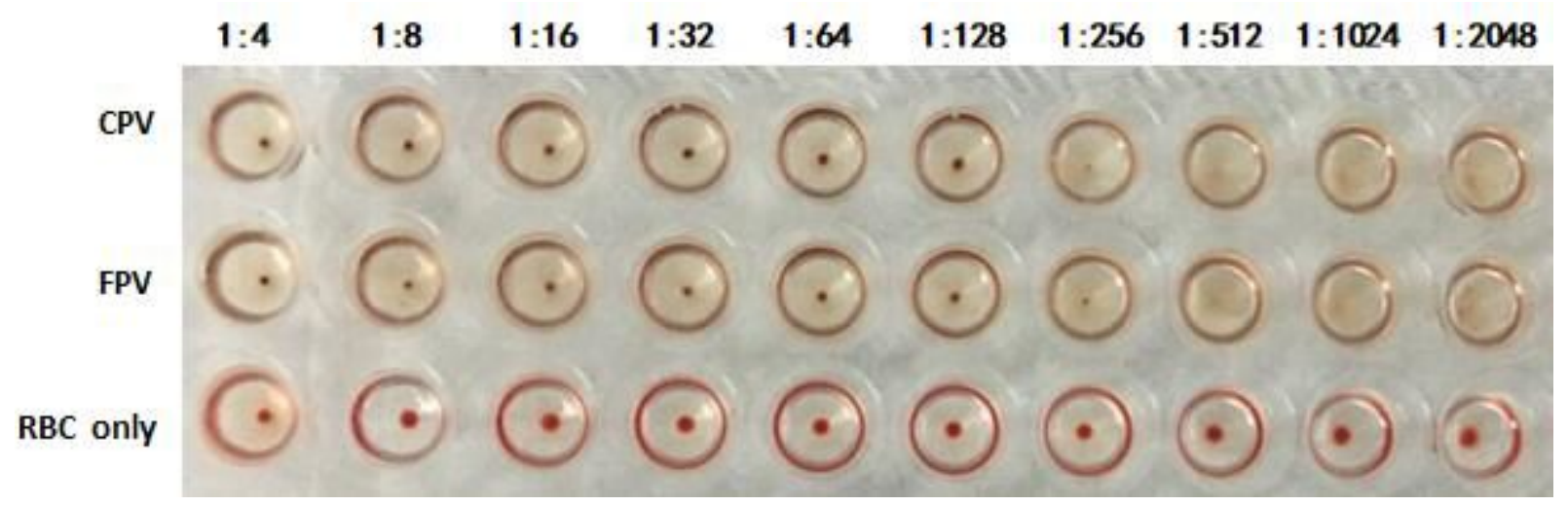

Figure 6

The hemagglutination test assay using isolated CPV-SH2001, FPV-SH2001 virus strain as antigen respectively.

\section{Control}

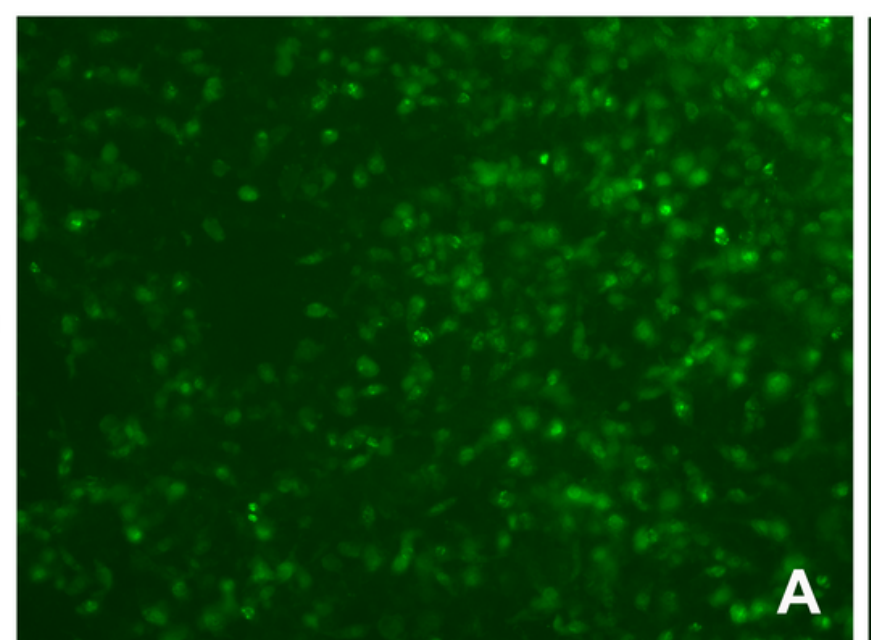

CPV SNT

\section{FPV SNT}

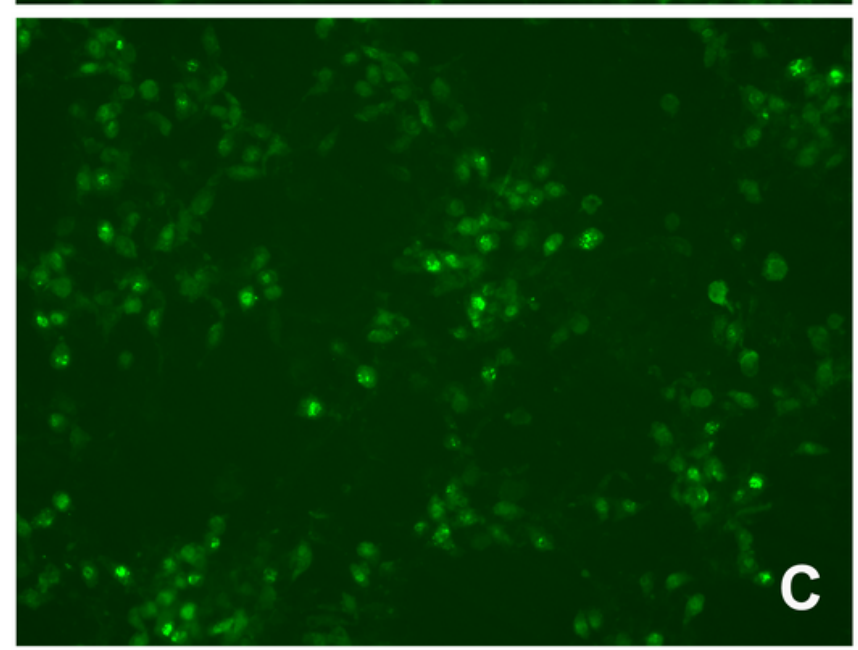

Neutralizing antibody (1:512 dilution)

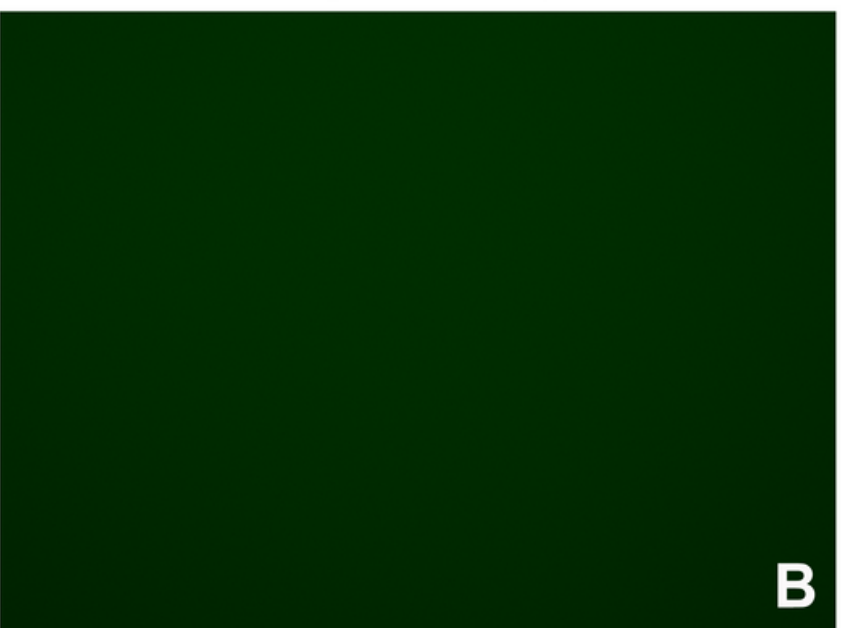

D

\section{Figure 7}

Both CPV-SH2001 and FPV-SH2001 virus strain were used to measure neutralizing antibody level induced by vaccine strain NL-35-D. A, CPV-SH2001 positive control; $B$, antibody 1:512 dilution against CPV- 
SH2001; C, FPV-SH2001 positive control; D, antibody 1:512 dilution against FPV-SH2001.

\section{Supplementary Files}

This is a list of supplementary files associated with this preprint. Click to download.

- originalgels.zip 